\title{
The individual health doctrine: some findings
}

\author{
Apanasenko Gennadii Leonidovich ${ }_{\mathrm{ABCDEFG}}$
}

Vincent Pol University, Poland

\section{Summary}

The article generalizes the author's findings of years of research. Methodology and a simple method of estimation of the level of health of individual were developed. It was established that the basis of somatic health depends on the effectiveness of the apparatus of the mitochondria that appeared on the organism's level in the form of maximum aerobic capacity of energy production. The increase of aerobic capacity of the biological systems causes two systems of the feedback that are the expansion of reserve and economization of functions. This finding was used to build the "scale" of individual health. The application of this method during population's studies revealed a number of new phenomena, such as the "safe level" of health, above which neither endogenous risk factors of coronary heart disease, nor manifestation of the disease, nor "self-development" of the pathological preventive rehabilitation process after an individual left the "safe zone" of health and others have not been registered. A new direction of the primary individual prevention of IHD (in addition to the population's and the group's prevention) was initiated. It was called "preventive rehabilitation" - return of the individual to a "safe zone" of health.

Keywords: individual health, chronic non-communicable diseases, ischemic heart disease, primary individual prevention, preventive rehabilitation.

\section{Introduction}

The major paradox of the modern medicine is having an objective to achieve health it deals with diseases instead. Year by year as the result of this paradox we are getting know more about diseases, diagnostic and treatment methods etc. But this way will never lead us to our goal to achieve health. For health is not an absence of a disease, and it is not an ephemeral "well-being", it is an individual's ability to perform its biological and social functions. In order to achieve health it is necessary to study phenomenon of health and its laws and use them to achieve health.

Medicine has been researching the problem of an individual health for more than two thousand years. The results were portrayed by Doll R. [1]: “There were many trails to measure positive health on a scale; however the measurement of positive health so far is the same illusion as the measurement of happiness, beauty, and love". Situation has been changed in recent years.

Since the times of Galen three main states of a human being have been determined: health, disease, and preexisting disease. Russian scientist Lazarev NV and his followers proved the existence of fourth state - the State of Nonspecifically Increased Resistibility (SNIR) when human not only feels healthy but also has at disposal certain "reserve of safety" that provides him the best conditions for survival. This state can be reached by means of different health improving methods such as natural substances which Lazarev NV called adaptogens.

The first publication of Lazarev NV devoted to SNIR was released in the Journal of Pathophysiology and Experimental Medicine [2]. The final analysis of the research works of Lazarev NV and his followers is the following: SNIR is characterized by two main particularities of an organism, the first one is expansion of functional reserve and the second one is economization of functions. The essential part of this process is in increase of power of intracellular generation of energy by improving of mitochondrial apparatus.

Lazarev's SNIR doctrine further became the ground for Theory of Valeology. It was the base for Lazarev's follower Brehman I.I. who was the first who introduced $[3,4]$ The Valeology Science and highlighted "individual health" category as a subject of the study. At the same time he devoted attention to philosophical issue and development of practicalities of use of adaptogens and its influence on a state of functions of healthy human instead of phenomenology of health. Phenomenology of health has been explored in our research $[5,6,7,8]$.

\section{Theory}

Having a certain theoretical and practical experience in the field of extreme medicine (time period before lapse into hypoxic coma after inhalation of a hypoxic mixture, the research of physical efficiency before and after massive blood loss, the dynamics of the professional performance of submarine operators under the monthlong influence of complex adverse environmental factors, etc.) we came to the conclusion that a common feature of the human organism to resist to adverse impacts does exist, and this potential energy of biological system (it corresponds to the second law of thermodynamics), depends on the oxidative capacity of the mitochondria. It is obvious that the main condition for the existence of life on Earth is the ability to absorb energy from the environment, accumulate and use it to implement the vi- 
tal processes. The higher available reserves of bioenergy are, the higher is vital capacity of an organism.

The ability to mobilize resources of organs, systems, and total organism is the first condition of immediate adaptation of an organism to extreme factors impact. And the more energy production per unit of body weight is the more effective is the biological function of survival. The ability to increase the absorption of oxygen when necessary determines the reserve of energy that can be used to intensify the vital processes. The higher the capacity is the higher is an organism's vitality. Hereby, the criterion that may become the basis for the "measurement" of health has been identified. On the organism's level, the energetic potential of biological system can be characterized by the maximal aerobic capacity of an organism that are power and efficiency of aerobic mechanisms of energy production. These findings were for the first time published in our article "The possibility of quantitative estimation of human health" [9]. More thorough theoretical and practical study of this issue was provided in the monograph "The Evolution of bioenergy and human health" [10].

\section{Materials and methods}

We have find a systemic feedback of an organism, that accompanies increasing of organism's resistance to various impact (based on the increasing energy potential of biological system, expanding physiological reserves and economization of functions), and we developed express-scale of a health level measurement. It includes simple indices of indicators of functions that reflect above mentioned patterns. It was found that the measurement of the health level, calculated by means of our express-system, has a high correlation coefficient with maximum oxygen consumption (0.806).

\section{Results}

Low work content, low price of use and availability for nurses staff of the express-scale of a health level measurement allowed us to perform thousands of experiments on healthy and sick individuals aged from 6 to 80 years. Our scientific findings made it possible to identify and describe new phenomena of an individual health:

- Individual health can be "measured" by direct indicators and therefore can be controlled (develop, maintain, restore);

- The higher level of health is, the lower probability of endogenous risk factors and manifested forms of ischemic heart disease (IHD);

- The "safe" level of health does exist, neither endogenous risk factors nor manifested forms of diseases can be defined above it;

- When an individual leaves the "safe zone" of health the phenomenon of "self-development" of a pathological process is observed;

- With increase of aerobic capacity of energy produc- tion the involution of endogenous factors of IHD risk occurs;

- The return of the individual to the "safe zone" of health can be interpreted as a "preventive rehabilitation".

Our research work also shows high correlation between the level of health (read - the function of mitochondria) and intensity, and also the combination of risk factors of IHD $(r=0,7-0,8)$. Hereby, we can assume that there is the main leading risk factor that causes IHD (and possibly chronic non communicable disease) and it is an insufficiency of mitochondrial function that goes beyond the limits defined by the laws of evolution. All other endogenous risk factors are consequences only.

\section{Discussion}

It is shown that the function of mitochondria that appears on the organism's level as maximum capability of the aerobic energy production is the criterion of the viability of an individual, in other words, the level of his health. This corresponds to the data on the most appropriate integral characteristic of biological age of an individual. After many years of systematic research that have been conducted at the Institute of Gerontology of Academy of Medical Sciences of Ukraine [11] the interrelation between the functional (biological) age and maximum oxygen consumption by an individual, which is described by a correlation coefficient of 0.840 for men and 0.813 for women was determined. Thus, the energetic potential of biological system actually reflects its viability and may serve as an integral criterion of viability and biological age.

Analysis of the literary sources and own information made it possible to substantiate assumption on direct cause of the epidemics of chronic non-communicable disease (NCD) that has swept the world in the second half of the $20^{\text {th }}$ century and become a main cause of death in the world today. It was proved that the cause is the mitochondrial insufficiency conditioned by a number of social and socio-hygiene factors including the modern lifestyle and environmental pollution. Thus, presumably the one common leading risk factor of NCD is insufficiency of mitochondrial function that goes beyond the limits defined by the laws of evolution [10].

After many thousands of studies of Ukrainian population it was discovered that currently only about $1 \%$ of the population is in the "safe" zone of health. This is the medical-biological basis of depopulation and accelerated aging nowadays (20 years ago, this rate was $8 \%)$.

The obtained data allowed ground a new trend in the health care that is the primary prevention of coronary heart disease of an individual (in addition to population and group strategies for primary prevention of this group of diseases). This is the way to prevent depopulation and accelerated aging on the former Soviet Union territory. It becomes obvious that the improvement of diagnostic and treatment in public health does not able to affect radically on morbidity and mortality 
rates, as pills do not treat low level of health. Only the adoption of the higher level of aerobic energy supply functions (performance of mitochondria) by vast part of the population may solve this problem (physical aerobic and hypoxic training, adaptogens, L-carnitine, some Eastern breathing techniques, etc.). All these data were explored in the monograph "Medical valeology" [6] and "Sanology. Basics of health management" [8].

The basics of the formation of NCD are laid in childhood and adolescence. In our monograph "The physical development of children and adolescents" [5], we pointed that the methodology currently used to solve the problem was wrong. The estimation of physical development performed according to regional standards of anthropometric indices does not give any information of the health and reflects only the results of probable level of sanitary and hygienic problems. Based on this data, as well as on known principle that growth and development of an organism is fulfilled by two reciprocal processes (increase in the protoplasmic mass and systemogenesis-organogenesis), we postulate the necessity for the measure of physical development (level of health) to be focused on systemogenesis processes as the basis of non-specific resistance of an organism. The measurement criteria are the same as in the measurement of adults' health - provision and economization of functions. The results of the measurement of physical development display the veritable position of an individual on the coordinate system "health-disease" $[6,8]$.

Accumulated findings on the issue of individual health (the original subject of the study was personal health, its definition with operational criteria, theoretical and methodological principles, methods for solving practical problems, etc.) helped to open a new applied specialty the "sanology therapeutist" and to prepare qualification characteristics (approved by Ministry of Health of Ukraine in 1991) and to create the concern department in P.L. Shupik National Medical Academy of Postgraduate Education with the purpose to train doctors of this specialty (1992). Our textbook "Sanology" for medical doctors was published in 2011 [7].

\section{Conclusions}

After years of research, the methodology and simple method of the measurement of the level of health of an individual were developed. It was also proved that the base of the somatic health derives from the effectiveness of the apparatus of the mitochondria that turned out in the form of maximum aerobic capacity of energy production on the organism's level. With an increase of aerobic potential of a biological system two system of feedback were discovered, one is expansion of reserve and second one is economization of functions. These facts were used to build the "scale" of individual health. The application of this method during population studies revealed a number of new phenomena, such as the "safe level" of health, above which neither endogenous risk factors of coronary heart disease, nor manifestation of the disease, nor "self-development" of the pathological process after an individual left the "safe zone" of health and others have not been registered. A new direction of the primary individual prevention of IHD (in addition to the population's and the group's prevention) was initiated. It was called "return of the individual to a "safe zone" of health" (preventive rehabilitation).

\section{References}

1. Doll R. Prevention: some future perspectives. Prev. med. 1978. 4:486-497.

2. Lazarev N.V., Liublina E.I., Rozin M.A. The state of heightened nonspecific resistibility. Pathophysiology and Experimental Medicine. 1959. 3: 16-21.

3. Brehman II. Philosophic and methodological aspects of human health. Philosophical matters. 1982. 2: 48-53.

4. Brehman II. Introduction to valeology - science of health. Science, Lvov. 1987.

5. Apanasenko G.L. The physical development of children and adolescents, Health, Kiev.1985.

6. Apanasenko G.L. Popova LA. Medical valeology. Health, Kiev. 1998.

7. Apanasenko G.L. and joint authors. Sanology (Course Textbook for Institutes of Higher Education of III-IV level of accreditation). Danylo Galytsky Lviv Medical University, Lviv. 2011.

8. Apanasenko G.L., Popova L.A., Maglevanyi A.V. Sanology. Basics of health management. Lambert Academic Publishing. 2012.

9. Apanasenko G.L. The possibility of quantitative estimation of human health. Hygiene and sanitary. 1985. 6: 55-58.

10. Apanasenko G.L. The evolution of bioenergy and human health. Petropolis, Saint Petersburg. 1992.

11. Chebotarev D.F., Korshunov I.T. Premature (accelerated) aging: causes, diagnostics, prevention and treatment. Medical world. 2001V.1, 1:28-38.

\section{Correspondence address:}

Professor Apanasenko Gennadii Leonidovich MD, Ph.D. Ukraine, Kiev-02068, Koshitsa str.10/21, ap. 39.

+38 067-7749437, +38 044 235-42-09

E-mail: apanas@ukr.net 